\title{
Value of Three-Dimensional Optical Coherence Tomography and Fundus Photochromy in Correlating the Fluorescein Leaking Sites of Acute Central Serous Chorioretinopathy
}

\author{
Guang-Hui Wang ${ }^{a}$ Jun Zhang ${ }^{a}$ Dan Zhang ${ }^{a}$ Fu-Li Lv ${ }^{a}$ Le-Xin Wang ${ }^{b}$ \\ aDepartment of Ophthalmology, Liaocheng People's Hospital and Liaocheng Clinical School of Taishan \\ Medical University, Liaocheng City, PR China; ${ }^{b}$ School of Biomedical Sciences, Charles Sturt University, \\ Wagga Wagga, N.S.W., Australia
}

\section{Key Words \\ Central serous chorioretinopathy · Optical coherence tomography $\cdot$ Photochromy $\cdot$ Fundus angiography}

identification rate of the leakage spots. The combination of the two noninvasive techniques may be used as an alternative diagnostic or evaluation tool for acute CSCR.

Copyright $\odot 2011$ S. Karger AG, Basel

\begin{abstract}
Objectives: This study was designed to investigate the value of 3-D optical coherence tomography (3-D OCT) combined with fundus photochromy in the diagnosis of acute central serous chorioretinopathy (CSCR). Subjects and Methods: 3-D OCT and fundus photochromy were performed on 30 patients (36 eyes) with acute CSCR. Fluorescein angiography (FA) was also performed to confirm the diagnosis and to obtain the fluorescein leakage sites. Results: 22 eyes presented neurosensory retinal detachment determined by 3-D OCT (28 leakage spots), 1 eye showed retinal pigment epithelium (RPE) detachment (2 leakage spots), and 13 eyes showed both neurosensory and RPE detachment (17 leakage spots). 3-D OCT showed significant changes in the RPE in 36 of 47 leakage spots (76.6\%). Fundus photochromy showed whitegray changes in 22 of the 47 leakage spots (59.6\%). 47 leakage spots were identified by FA in 36 eyes. The combination of 3-D OCT and fundus photochromy identified 42 of the 47 leakage spots (89.4\%) spotted by FA. Conclusion: A combination of 3-D OCT and fundus photochromy offered a high
\end{abstract}

\section{Introduction}

Central serous chorioretinopathy (CSCR) is a chorioretinal disease commonly seen in young and middleaged men. Patients often develop one or more small areas of retinal pigment epithelium (RPE) serous detachment in the macula or in the paramacular area $[1,2]$. The gold standard for CSCR diagnosis is fluorescein angiography (FA), which displays subretinal fluid accumulation, RPE detachment and typical dye leakage from the choroid into the subretinal space [1].

Optical coherence tomography (OCT) is a noninvasive diagnostic technique that uses reflection of light off the retinal layers to create a false-color tomographic image of the retina and the RPE [2]. OCT provides highresolution retinal images in vivo [3]. It has been used to study the anatomy and pathogenesis of various ocular disorders affecting the posterior segment. Three-dimensional (3-D) OCT can generate high-definition 3-D imag-

\section{KARGER}

Fax +4161306 1234

E-Mail karger@karger.ch

www.karger.com
(C) 2011 S. Karger AG, Basel

$1011-7571 / 11 / 0203-0283 \$ 38.00 / 0$

Accessible online at:

www.karger.com/mpp
Prof. Le-Xin Wang

School of Biomedical Sciences

Charles Sturt University

Wagga Wagga, NSW 2678 (Australia)

Tel. +61 26933 2905, Fax +61 26933 2587, E-Mail lwang@ csu.edu.au 

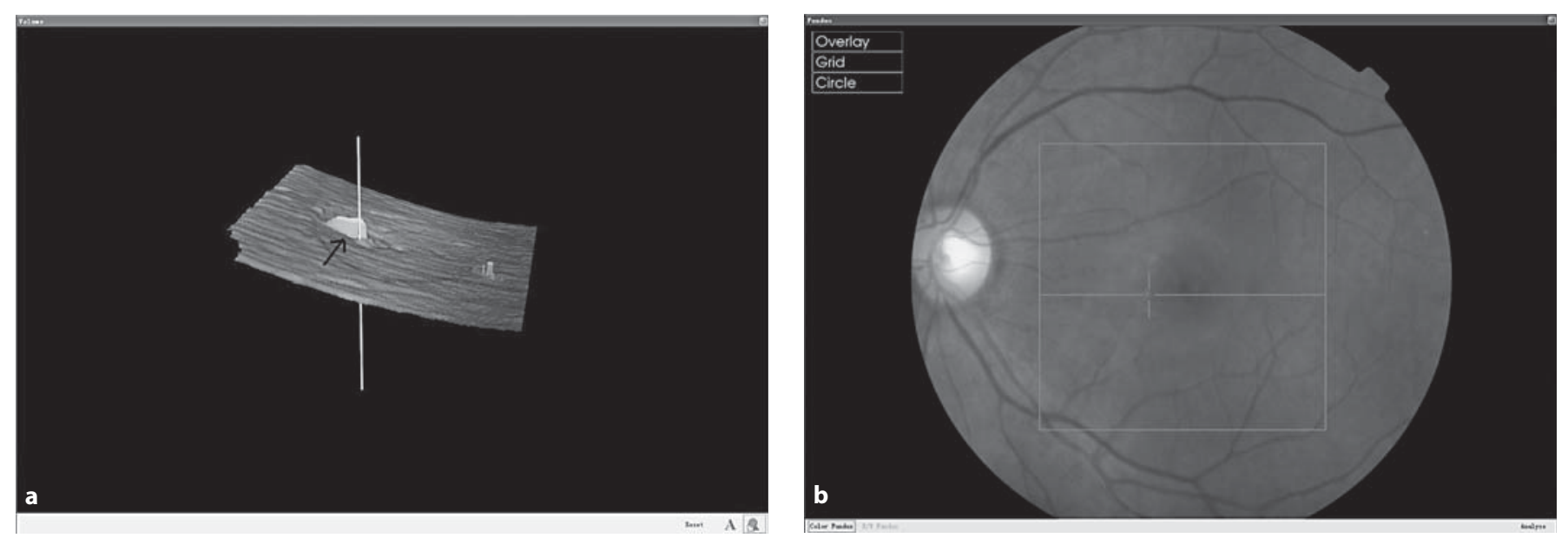

Fig. 1. 3-D OCT in acute forms shows bulges protruding from the RPE layer. a Apophysis (arrow) of RPE on 3-D OCT. b White-gray changes on fundus photochromy.

ing, using high-speed and ultrahigh-resolution OCT [4]. This technique improves image quality, retinal coverage and structural registration [4]. The purpose of this study was to investigate the value of 3-D OCT in the identification of retinal lesions in acute CSCR.

\section{Subjects and Methods}

This study was approved by the institutional review board of Liaocheng People's Hospital. Informed consent was obtained from all participants before the study. Between March and July 2008, 30 patients ( 24 males and 6 females aged $24-55$ years; median: 41 years) were recruited to this study. Among these patients, 36 eyes (18 left eyes and 18 right eyes) were affected by acute CSCR. The duration of the CSCR was 3-60 days. All patients underwent visual acuity testing and fundus examination.

3-D OCT (OCT-1000; Topcon Corp., Japan) was performed following FA (IMAGEnet 2000, Topcon TRC-50IX; Topcon) on the same day. Horizontal and vertical OCT scans of the fovea and fluorescein leakage sites were obtained. The speed of the OCT scan was selected at $27,000 / \mathrm{s}$ with an axial differential of $5 \mu \mathrm{m}$. The field of each scan was $6 \times 6 \mathrm{~mm}$, with a scan depth of $2.3 \mathrm{~mm}$. OCT scans were repeated 3 times to ensure reproducibility.

Fundus photochromy was performed on the same day, prior to or immediately after the 3-D OCT scan. The characteristics of the leakage sites detected by OCT and fundus photochromy were compared with the findings by FA to determine the mutual relation and the nature of the findings. Two experienced ophthalmologists (G.H.W. and J.Z.) performed these fundus examinations. In the event of significant differences in findings between the two investigators, a third ophthalmologist's opinion was sought to reach an agreement. The OCT findings were classified into 3 categories: (1) serous neurosensory, (2) isolated RPE, and (3) RPE and neurosensory detachments.

A descriptive statistical analysis was performed using SPSS software version 11.5. Data are expressed as means \pm SD. Categorical data were analyzed by the $\chi^{2}$ test where appropriate. $\mathrm{p}<$ 0.05 was considered statistically significant.

\section{Results}

Visual acuity was $0.1-0.3$ in 2 eyes, $0.4-0.6$ in 14 eyes, $0.7-0.9$ in 16 eyes, and over 0.9 in 4 eyes. FA showed 1 single leakage site in 30 eyes, and more than 2 leakage sites in 6 eyes. Overall, there were 47 leakage sites in the 36 eyes. Thirty-eight leakage sites were pinpoint, and 9 were round or irregular in shape.

The 3-D OCT scans showed foveal distortion and cystic and atrophic macular changes in all eyes. Serous neurosensory detachments were found in 28 leakage sites (59.6\%) from 22 eyes (61.1\%), 17 of them showing RPE elevation with fragmented reflection zones and a localized detachment of the neurosensory retina, with a poor reflection zone between the two epithelial layers (fig. 1). Isolated RPE detachment was detected in 2 leakage sites from 1 eye; RPE and neurosensory detachments (fig. 2) were found in 17 leakage sites (36.2\%) from 13 eyes (36.1\%). The RPE detachment was located within the neurosensory detachment area.

Of 47 leakage sites (76.6\%) identified by FA, 3-D OCT identified 36 (37.5\%) as typical RPE detachment or as arched apophysis. Fundus photochromy identified 28 

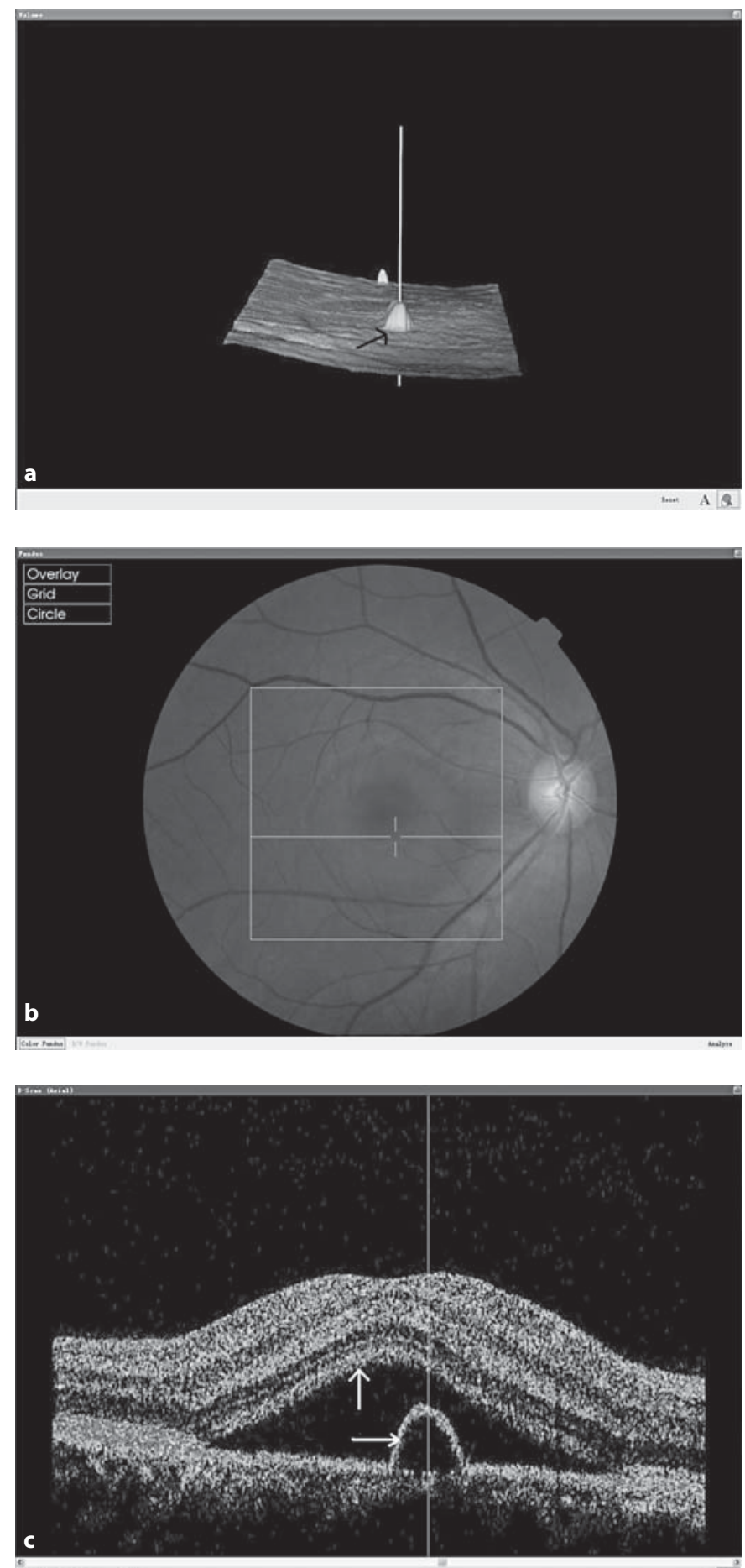

ine the the

$A \sqrt{2}$

Fig. 2. Neurosensory and RPE detachment. a Arched apophysis (arrow) on 3-D OCT. b White-gray changes on fundus photochromy. c Neurosensory and RPE detachment (arrows).
(59.6\%) of the 47 leakage sites. There was no significant difference in identification rate between 3-D OCT and fundus photochromy $(\mathrm{p}>0.05)$.

Six leakage sites (12.8\%) were identified by fundus photochromy but not by 3-D OCT, but 14 sites (29.8\%) identified by 3-D OCT were not identified by fundus photochromy. Overall, 5 leakage sites (10.6\%) were neither identified by 3-D OCT nor fundus photochromy. The combination of 3-D OCT and fundus photochromy had a correct identification rate of $89.4 \%$.

\section{Discussion}

CSCR is characterized by neurosensory retina or RPE detachment that is commonly unilateral but can also be bilateral $[1,2]$. Laser photocoagulation has been widely used with CSCR to prevent recurrence and to facilitate recovery. In recent years, photodynamic therapy has been emerging as a potential treatment for CSCR $[5,6]$. Both laser photocoagulation and photodynamic therapies require regular fundus examination and assessment of the leakage sites. Although FA is more accurate in assessing the retinal lesions, the invasiveness of this technique may hinder its repeated use in the long term. Therefore, noninvasive diagnostic systems such as OCT or photochromy may be used as alternative diagnostic or investigative tools.

Hirami et al. [7] compared the findings by OCT and FA in 30 eyes. They found that 22 of 25 eyes with acute CSCR showed OCT abnormalities in the RPE (88\%). These abnormalities included RPE detachment in 9 eyes (36\%) and a small bulge on the RPE at the posterior pole in 15 eyes (60\%). Of the 29 eyes with acute or chronic CSCR, FA showed characteristic pinpoint leakage in 20 eyes (69\%) [7]. However, it was unclear if the FA findings were matched with those from the OCT scans [7]. Another recent study on Fourier domain OCT examination revealed that the new OCT scan can reveal a leakage site through which fluid may pass to the subretinal area [8]. The authors concluded that Fourier domain OCT may offer new information to facilitate understanding of the mechanisms of acute CSCR [8].

Our study showed that 3-D OCT can identify $76.6 \%$ of the leakage sites. Fundus photochromy was able to identify $59.6 \%$ of the sites. If the two techniques are used concurrently, $89.4 \%$ of the leakage sites can be discovered. These data indicate that the two noninvasive techniques may be used in combination for the diagnosis or followup of CSCR. It must be noted that $10.6 \%$ of the leakage 
sites were discovered neither by 3-D OCT nor by photochromy. The features or characteristics of these misdiagnosed leaking sites require further investigation in order to improve the diagnostic accuracy of 3-D OCT and photochromy.

The major findings of this study are: (1) The vast majority of acute CSCR were caused by detachment of the neurosensory epithelium or RPE, or by detachment of both. (2) 3-D OCT appeared to have a reasonably correct identification rate for the leakage sites at various stages of acute CSCR. (3) Combination of 3-D OCT and fundus photochromy increased the correct identification rate for leakage sites to $89.4 \%$. These noninvasive measures can be readily applied, and are effective in monitoring followup, and especially the clinical effect after any form of treatment, without repeating FA.

\section{Conclusion}

A combination of 3-D OCT and fundus photochromy offered a high identification rate of the leakage spots. The combination of the two noninvasive techniques may be used as an alternative diagnostic or evaluation tool for acute CSCR.

\section{References}

- Kitaya N, Nagaoka T, Hikichi T, Sugawara R, Fukui K, Ishiko S, Yoshida A: Features of abnormal choroidal circulation in central serous chorioretinopathy. Br J Ophthalmol 2003;87:709-712.

$\checkmark 2$ Montero JA, Ruiz-Moreno JM: Optical coherence tomography characterisation of idiopathic central serous chorioretinopathy. $\mathrm{Br}$ J Ophthalmol 2005;89:562-564.

3 Baumal CR: Clinical applications of optical coherence tomography. Curr Opin Ophthalmol 1999;10:182-188.
4 Srinivasan VJ, Wojtkowski M, Witkin AJ, Duker JS, Ko TH, Carvalho M, Schuman JS, Kowalczyk A, Fujimoto JG: High-definition and 3-dimensional imaging of macular pathologies with high-speed ultrahigh-resolution optical coherence tomography. Ophthalmology 2006;113:2054e1-e14.

$\checkmark 5$ Cardillo Piccolino F, Eandi CM, Ventre L, Rigault de la Longrais RC, Grignolo FM: Photodynamic therapy for chronic central serous chorioretinopathy. Retina 2003;23: 752-763.

6 Chan WM, Lai TY, Lai RY, Liu DT, Lam DS: Half-dose verteporfin photodynamic therapy for acute central serous chorioretinopathy: one-year results of a randomized controlled trial. Ophthalmology 2008; $115: 1756-$ 1765 .
7 Hirami Y, Tsujikawa A, Sasahara M, Iwama D, Yoshimura N: Alterations of retinal pigment epithelium in central serous chorioretinopathy. Clin Exper Ophthalmol 2007;35: 225-230.

8 Fujimoto H, Gomi F, Wakabayashi T, Sawa M, Tsujikawa M, Tano Y: Morphologic changes in acute central serous chorioretinopathy evaluated by Fourier-domain optical coherence tomography. Ophthalmology 2008;115:1494-1500. 